\title{
Psicopatía y adicciones en mujeres: Estudio comparativo utilizando el Psychopathy Checklist Revised y el Comprehensive Assessment of Psychopathic Personality
}

\section{Psychopathy, addictions and female gender: Comparative study using the Psychopathy Checklist Revised and the Comprehensive Assessment of Psychopathic Personality}

\author{
Gerardo Flórez****a, Ventura Ferrer**, Luis S. García**, María R. Crespo**, \\ Manuel Pérez*, Pilar A. Sáiz***************. \\ * Centro de Investigación Biomédica en Red de Salud Mental (CIBERSAM), Oviedo. España. \\ ** Servicios Médicos del Centro Penitenciario de Pereiro de Aguiar, Ourense. España. \\ *** Departamento de Psiquiatría. Universidad de Oviedo. España. \\ **** Servicio de Salud del Principado de Asturias (SESPA). España. \\ ***** Instituto de Investigación Sanitaria del Principado de Asturias (ISPA). España.
}

\section{Resumen}

La investigación en psicopatía se ha centrado tradicionalmente en la evaluación de varones con el Psychopathy Checklist Revised (PCL-R). La investigación en mujeres utilizando otros instrumentos de evaluación es muy escasa. El objetivo de este estudio es evaluar la psicopatía, utilizando varios instrumentos, es una muestra que incluya mujeres y varones. 204 internos (edad media $(\mathrm{SD})=40,93(11,8))$, 28 mujeres $(13,7 \%)$, del Centro Penitenciario de Pereiro de Aguiar (Ourense) participaron en este estudio. Se recogieron variables sociodemográficas, consumo de sustancias,antecedentes delictivos y fueron evaluados con los siguientes instrumentos: PCL-R, Comprehensive Assessment of Psychopathic Personality (CAPP) y el International Personality Disorder Examination. En esta muestra representativa de la población penitenciaria se observó que en el PCL-R los varones obtenían puntuaciones significativamente más elevadas en la faceta 4 que mide la conducta antisocial. Las mujeres obtuvieron puntuaciones significativamente más elevadas en el CAPP en el dominio Self, que mide la conducta narcisista. No se obtuvo ningún síntoma o ítem que permitiese discriminar claramente a las mujeres psicopáticas de los varones psicopáticos utilizando un modelo de Máquinas de Vectores Soporte. El constructo de la psicopatía es similar para las mujeres y los hombres en esta muestra. Las mujeres presentan puntuaciones más elevadas de narcisismo y los varones de antisocialidad. Para valorar mejor estas diferencias psicopatológicas es mejor combinar el PCL-R con otro instrumento como el CAPP. Ningún síntoma o ítem tiene una puntuación que pueda recomendarse como método que permita discriminar a las mujeres psicopáticas de los varones psicopáticos.

Palabras clave: Psicopatía; mujer; adicciones; Psychopathy Checklist Revised; Comprehensive Assessment of Psychopathic Personality.

\begin{abstract}
Traditionally, psychopathy research has focused on assessing men with the Psychopathy Checklist Revised (PCL-R). Research on women with other assessment tools is scarce. The objective of this study is to evaluate psychopathy, using various tools, in a sample with both women and men. The study involved 204 inmates (mean age (DS) $=40.93$ (11.8)), 28 women (13.7\%), in the Pereiro de Aguiar penitentiary (Ourense). Sociodemographic, substance use, and criminal variables were collected, and all were evaluated with the following tools: PCL-R, Comprehensive Assessment of Psychopathic Personality (CAPP), and the International Personality Disorder Examination. In this sample, when assessed with the PCL-R, males obtained significantly higher scores on facet 4, which measures antisocial behaviour. Women obtained significantly higher scores on the Self domain in the CAPP, measuring narcissism. No symptom or item was able to clearly discriminate psychopathic women from psychopathic men in a Support Vector Machine model. The construct of psychopathy is similar for women and men in this representative penitentiary sample. Women showed higher scores for narcissism and men for antisociality. It is better to combine the PCL-R with another tool such as the CAPP to assess these psychopathological differences. No symptom or item has a score that can be recommended as a method for discriminating psychopathic women from psychopathic men.

Keywords: Psychopathy; women; addictions; Psychopathy Checklist Revised; Comprehensive Assessment of Psychopathic Personality.
\end{abstract}

Recibido: Marzo 2020; Aceptado: Noviembre 2020.

Enviar correspondencia a:

Gerardo Flórez. Francisco Huertas n ${ }^{\circ} 16^{\circ}$ A 32004 Ourense, España.

E-mail: gerardof@mundo-r.com 


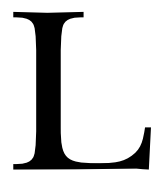

a psicopatía, o trastorno psicopático de la personalidad, se caracteriza por la presencia de los siguientes rasgos: baja respuesta emocional y ausencia de empatía, estilo interpersonal arrogante y grandioso y una conducta errática e impulsiva (Blackburn, 2007; Cooke y Logan, 2015). Sin embargo, no existe ningún síntoma / signo, o combinación de los mismos que sea patognomónico (Cooke y Logan, 2015). A pesar de las dificultades diagnósticas inherentes al diagnóstico de la psicopatía a través de entrevistas semi-estructuradas que miden la presencia de determinados síntomas o conductas, numerosos estudios relacionan la psicopatía con una mayor presencia de conductas antisociales, comisión de delitos, especialmente violentos, y una mayor tendencia a la recidiva en estas conductas y delitos (Blair y Lee, 2013; Cale, Lussier, McCuish y Corrado, 2015). Es por esto, que su valoración es fundamental en ámbitos clínicos y forenses, a la hora de establecer planes de seguimiento $\mathrm{y}$ tratamiento de pacientes o internos que se hayan visto implicados en conductas antisociales, y en especial hayan cometido delitos violentos.

A pesar de que la mayor parte de los estudios sobre psicopatía se han realizado en varones ingresados en centros penitenciarios, no existe, a priori, ningún motivo para pensar que las mujeres no puedan presentar este trastorno. De hecho, se estima que hasta un $17,4 \%$ de las mujeres que cometen actos violentos presentan un trastorno psicopático, frente a un $31 \%$ de los varones (Carabellese et al., 2019; Gray y Snowden, 2016). Estos datos ya permiten establecer que las mujeres presentan una prevalencia inferior para este trastorno, al igual que para otras patologías externalizadoras (Rial et al., 2019). Por otra parte, numerosos autores han indicado que existen diferencias en la forma en que se manifiesta la psicopatía en las mujeres, comparado con los hombres (Carabellese et al., 2019; Dolan y Vollm, 2009; Gray y Snowden, 2016; Guay, Knight, Ruscio y Hare, 2018; Thomson, 2017; Verona, Bresin y Patrick, 2013; Wynn, Hoiseth y Pettersen, 2012). Dado que la principal herramienta diagnóstica de este trastorno, el Psychopathy Checklist Revised (PCL-R), determina la presencia o ausencia de este trastorno a través de un punto de corte cuantitativo, no es difícil establecer que hombres y mujeres alcancen ese punto de corte a través de patrones sintomáticos cualitativamente distintos, y por lo tanto no deba extrapolarse toda la investigación en psicopatía realizada en varones a las mujeres. Es necesario comprobarlo a través de investigación rigurosa. Hasta la fecha, los estudios realizados indican que las mujeres obtienen puntuaciones más bajas en el PCL-R, sobre todo en los ítems relacionados con alteraciones de conducta, consumo de sustancias y adicciones, y antisocialidad. Además, la capacidad predictiva del diagnóstico de psicopatía en lo que se refiere a la comisión de nuevos delitos, especialmente de tipo violento, parece reducida en las mujeres con respecto a los hom- bres (Carabellese et al., 2019; Dolan y Vollm, 2009; Gray y Snowden, 2016; Guay et al., 2018; Nicholls, Ogloff, Brink y Spidel, 2005; Thomson, 2017; Verona et al., 2013; Wynn et al., 2012).

Por otro lado, el PCL-R ha sido criticado por poner un excesivo énfasis en las conductas criminales (Blackburn, 2007; Cooke, Michie, Hart y Clark, 2004). Este hecho tendría una gran importancia a la hora de valorar la presencia de psicopatía en mujeres, ya que estas presentan un patrón criminal diferente, con un menor consumo de sustancias y por lo tanto una menor incidencia de adicciones y los problemas derivados de las mismas. En este patrón, como ya se ha indicado previamente, la delincuencia juvenil, la revocación de la libertad condicional, las conductas antisociales y los delitos violentos serían menos frecuentes en mujeres (Carabellese et al., 2019; Dolan y Vollm, 2009; Gray y Snowden, 2016; Guay et al., 2018; Nicholls et al., 2005; Thomson, 2017; Verona et al., 2013; Wynn et al., 2012). Dado que todas estas conductas influyen en las puntuaciones del PCL-R no es difícil intuir el problema. Por lo tanto, ¿presentan las mujeres una prevalencia inferior en lo que a la psicopatía se refiere, con un patrón sintomático distinto al de los varones?, o, por el contrario, ¿se trata simplemente de un problema del instrumento de valoración y sus puntos de corte? Puntos de corte que han sido cuestionados por las diferencias culturales encontradas (Cooke y Michie, 1999; Cooke, Michie, Hart y Clark, 2005).

Una forma de resolver este problema es el uso combinado del PCL-R con otro instrumento de evaluación que excluya la conducta criminal en su valoración. El Comprehensive Assessment of Psychopathic Personality (CAPP) es la herramienta perfecta para esta valoración, ya que fue diseñada para ser capaz de evaluar la psicopatía sin valorar la criminalidad. Hasta la fecha ha demostrado ser un instrumento válido y robusto a través de diferentes estudios internacionales (Cooke, Hart, Logan y Michie, 2012; Florez et al., 2015; Sellbom, Cooke y Hart, 2015).

Por lo tanto, para investigar la presencia y características de la psicopatía en mujeres en comparación con los hombres, sería de gran utilidad comparar los resultados del PCL-R y el CAPP en una muestra representativa de internos de un Centro Penitenciario, con un consumo de sustancias similar para ambos géneros. Este es, precisamente, el objetivo del presente estudio.

\section{Material y métodos}

El Centro Penitenciario de Pereiro de Aguiar (Ourense) fue la institución donde se realizó el presente estudio. Entre abril de 2014 y abril de 2016 se ofreció la posibilidad de participar a todos los internos penados del Centro. Los criterios de inclusión fueron: haber cumplido por lo menos 6 meses de la condena en el Centro y firmar el consentimiento informado. Los criterios de exclusión fueron: 
no dominar el español de forma fluida y presentar una enfermedad orgánica (que implicasen dificultades manuales, visuales o del lenguaje significativas) o psiquiátrica (Trastornos Psicóticos, Trastornos Afectivos, Trastornos de Ansiedad y Demencia) que no permitiese al interno participar en el estudio.

Los datos de participación en el estudio fueron los siguientes: de los 330 internos evaluados, 204 cumplían los criterios de inclusión - exclusión y participaron en el estudio. Los 126 restantes no cumplían criterios por las siguientes causas: 10 no quisieron participar, 16 no dominaban de forma fluida el español, 32 presentaban una enfermedad orgánica o psiquiátrica que no les permitía participar, y 68 $(53,99 \%)$ internos no habían cumplido al menos 6 meses de su condena en el Centro. De estos 126, 11 (8,73\%) eran mujeres y el resto $115(91,27 \%)$ eran varones. La muestra final resulta representativa de la población del Centro Penitenciario.

De los participantes 176 eran varones, y 28 mujeres. Un dato del todo normativo, ya que en los Centros Penitenciarios españoles la proporción es de 12 hombres por cada mujer (Vicens et al., 2011).

La investigación fue aprobada por el Comité Ético de Vigo - Ourense - Pontevedra (2014/ 009). El estudio se realizó de acuerdo a la Declaración de Helsinki (World Medical, 2013).

Todos los participantes fueron evaluados siguiendo el siguiente protocolo:

- International Personality Disorder Examination (IPDE) versión DSM IV (Lenzenweger, Lane, Loranger y Kessler, 2007): Entrevista semi-estructurada destinada a realizar diagnósticos de trastornos de la personalidad (TP) categoriales siguiendo el modelo del DSM IV.

- Psychopathy Checklist Revised (PCL-R) (Hare, Clark, Grann y Thornton, 2000): Es la herramienta gold-standar en el campo de la evaluación de la psicopatía. Entrevista semi-estructurada, que apoyada por la información disponible en el historial y la que puedan aportar informadores que conozcan al evaluado, mide la psicopatía a través de la vida utilizando 20 síntomas. Cada síntoma se puntúa del 0 al 2. Su uso está adaptado al medio forense y penitenciario. Habitualmente, los síntomas se agrupan en un modelo estructural de 2 factores y 4 facetas: factor 1 interpersonal y afectivo [interpersonal (faceta 1), afectivo (faceta 2)] y un factor 2 de desviación social [estilo de vida (faceta 3), conducta antisocial (faceta 4)].

- Comprehensive Assessment of Psychopathic Personality (CAPP) (Kreis, Cooke, Michie, Hoff y Logan, 2012): Entrevista semi-estructurada en la que no se puntúa la criminalidad al contrario que en el PCL-R. Se valoran 33 síntomas, que se puntúan del 1 al 7 , y se estructuran en 6 dimensiones: apego, conductual, cognitivo, dominancia, emocional y sí mismo/self.
Los síntomas se evalúan en un periodo previo de 6 a 12 meses. Además de la entrevista, se recoge toda la información disponible a través del historial y de informadores que conozcan al interno desde hace, por lo menos, 6 meses.

- Protocolo "Ad hoc" de variables sociodemográficas, de consumo de sustancias y penitenciarias: género, edad, nacionalidad, años de educación completados, estado civil, estancia total en prisión en meses, tipo y número de delitos cometidos, tipo, edad de inicio y número de sustancias adictivas distintas de la nicotina consumidas a lo largo de la vida incluyendo aquellas que fueron prescritas como tratamiento (Metadona, Buprenorfina, Benzodiacepinas), separando el consumo de alcohol del abuso de alcohol.

G. Flórez valoró a todos los participantes usando el IPDE, el PCL-R y el CAPP, en una situación de ciego frente a los resultados de las variables sociodemográficas y penitenciarias.

\section{Análisis de datos}

Todo el análisis estadístico fue ejecutado con el software $\mathrm{R}$, versión 3.4.3. Se realizó una matriz de correlaciones con las puntuaciones totales del PCL-R, sus factores y facetas, y con las puntuaciones totales y los dominios del CAPP. Se realizó para toda la muestra, y para mujeres y varones por separado.

La descripción de las variables del estudio se elaboró a través de su media y desviación estándar en el caso de las variables continuas, y por su número de ocurrencias y porcentaje en las categóricas. En el caso de las variables continuas, las comparaciones entre dos grupos, al no presentar éstas normalidad, se hicieron aplicando el test de Mann-Whitney. Para las variables categóricas, las comparaciones se hicieron por medio del test de Chi-cuadrado, o bien el test exacto de Fisher en aquellos casos en los que las frecuencias teóricas eran inferiores a 5. Se usó el coeficiente de correlación de Pearson como método para medir la fuerza de asociación lineal entre variables continuas y modelos de regresión lineal múltiple y logística con el fin de determinar las posibles relaciones multivariantes existentes. En los modelos de regresión logística se utilizó como variable dependiente el género. La bondad de los modelos obtenidos se verificó con la ayuda del test de Hosmer y Lemeshow. Se descartó la presencia de problemas de multicolinealidad entre los predictores antes de su inclusión en los modelos de regresión logística.

Finalmente se aplicó un modelo de Máquinas de Vectores Soporte, cuyo objetivo es trazar de una forma computacionalmente eficiente hiperplanos capaces de realizar separaciones en espacios de características con un número elevado de dimensiones. En este estudio el objetivo de aplicar este modelo era determinar si resulta posible diferen- 
ciar entre hombres y mujeres en función de los resultados obtenidos en los instrumentos CAPP y PCL-R. En aquellos casos en los que los datos no sean linealmente separables, como es el problema que se plantea en esta muestra, éstos se pueden transformar a un espacio de más alta dimensión en el que resulte posible hacer una separación lineal de los mismos. Otra posible estrategia, consiste en aceptar que habrá datos que no puedan ser correctamente clasificados, y tratar de clasificar correctamente la mayor cantidad de información a través de la minimización de su función de pérdida. Una combinación de ambas técnicas será la que se emplee en este trabajo.

Se consideró significativo un valor de $\mathrm{p}<0,05$.

\section{Resultados}

En la Tabla 1 se presentan la distribución de estas variables y la prevalencia de los TP según el IPDE en función del género.

Tabla 1. Variables sociodemográficas y penitenciarias de la

muestra, junto a la prevalencia en la misma de Trastornos de la Personalidad según el IPDE, en función del género.

\begin{tabular}{|c|c|c|c|c|c|c|}
\hline \multirow{2}{*}{$\begin{array}{l}\text { Variables } \\
\text { Edad [media (DS)] }\end{array}$} & \multirow{2}{*}{\multicolumn{2}{|c|}{$\begin{array}{c}\text { Mujeres (28) } \\
40,89(11,16)\end{array}$}} & \multirow{2}{*}{\multicolumn{2}{|c|}{$\begin{array}{c}\text { Hombres (176) } \\
40,97(11,20)\end{array}$}} & \multicolumn{2}{|c|}{$\mathbf{p}$} \\
\hline & & & & & $t=0,03$ & $p=0,97$ \\
\hline $\begin{array}{l}\text { Educación años completados } \\
\text { [mediana, (DS)] } \\
\text { Básica } \\
\text { Superior } \\
\end{array}$ & $\begin{array}{l}8,75 \\
0,21 \\
\end{array}$ & $\begin{array}{l}(2,15) \\
(0,69) \\
\end{array}$ & $\begin{array}{l}8,84 \\
0,24 \\
\end{array}$ & $\begin{array}{l}(1,95) \\
(0,88) \\
\end{array}$ & $\begin{array}{l}t=0,20 \\
t=0,17\end{array}$ & $\begin{array}{l}p=0,84 \\
p=0,86\end{array}$ \\
\hline $\begin{array}{l}\text { Estado civil } \\
\text { Casado } \\
\text { Casado } 2^{a} \text { nupcias } \\
\text { Viudo } \\
\text { Separado } \\
\text { Divorciado } \\
\text { Soltero }\end{array}$ & $\begin{array}{l}5 \\
2 \\
1 \\
6 \\
5 \\
9\end{array}$ & $\begin{array}{l}17,86 \% \\
7,14 \% \\
3,57 \% \\
21,43 \% \\
17,86 \% \\
32,14 \%\end{array}$ & $\begin{array}{l}28 \\
14 \\
0 \\
17 \\
33 \\
84\end{array}$ & $\begin{array}{l}15,91 \% \\
7,95 \% \\
0,00 \% \\
9,66 \% \\
18,75 \% \\
47,73 \%\end{array}$ & $\begin{array}{l}X^{2}=3,83 \\
X^{2}=0,01 \\
X^{2}=2,36\end{array}$ & $\begin{array}{l}p=0,79 \\
p=1 \\
p=0,1373 \\
p=0,05 \\
p=0,91 \\
p=0,12\end{array}$ \\
\hline $\begin{array}{l}\text { Meses totales en prisión [media } \\
\text { (DS)] }\end{array}$ & 45,61 & $(43,27)$ & 79,77 & $(87,46)$ & $t=3,25$ & $p<0,01$ \\
\hline
\end{tabular}

Nota. DS: Desviación estándar; IPDE: International Personality Disorder Examination.

Dicha Tabla 1 pone de manifiesto que ambos grupos eran similares, ya que sólo se encontraron diferencias estadísticamente significativas en las siguientes variables: los varones habían pasado más tiempo en centros penitenciarios, habían consumido y abusado más del alcohol por lo que presentaban un porcentaje superior de condenados por conducir bajo los efectos del alcohol, y también habían consumido más cannabis; los varones habían recibido más diagnósticos de trastorno antisocial de la personalidad (TAP), mientras que las mujeres habían recibido más diagnósticos de los siguientes TP: borderline, narcisista, histriónico y dependiente.

La matriz de correlaciones (Tabla 2), debe ser analizada desde la perspectiva de los patrones que surgen en cada grupo a la hora de comparar los resultados, y no desde un punto de vista de las meras puntuaciones, ya que al ser el grupo de mujeres más reducido sus puntuaciones son menores por un efecto de restricción del rango. En esta tabla se observa como los patrones de correlación de la muestra total se mantienen al separar la muestra en varones y mujeres. Las facetas del PCL-R que forman los factores correlacionan de forma potente entre ellas (facetas 1 y 2 , versus 3 y 4), pero de una forma débil con las facetas del otro factor. Destaca en mujeres la elevada correlación entre las facetas 1 y 2. Como era de esperar la faceta 1 (interpersonal) consigue las mejores puntuaciones de correlación con los dominios de CAPP de Dominancia y Self, y sus correlaciones más débiles son con Conductual y Cognitivo para ambos grupos. Con respecto a la faceta 2 (afectivo), y también para ambos grupos, las puntuaciones indican una mejor correlación con Apego, Dominancia, Emocional y Self, y de nuevo las más bajas serían con Conductual y Cognitivo. En este grupo de correlaciones destaca la relación en el grupo de mujeres entre la faceta 2 del PCL-R y los dominios de Apego y Self del CAPP. Para la faceta 3 (estilo de vida), se aprecia en ambos grupos una correlación potente con la dimensión Conductual, otra algo menos intensa con Cognitivo, y correlaciones poco potentes con el resto de las dimensiones del CAPP. El mismo patrón se observa para la faceta 4 (conducta antisocial). Con respecto a las dimensiones del CAPP, Apego, correlaciona de forma potente en ambos grupos, con Dominancia, Emocional y Self. Conductual y Cognitivo correlacionan bien entre ellos para ambos grupos, y en los varones, pero no en las 
mujeres, correlacionan bien con el resto de dimensiones. Dominancia se erige como la dimensión fundamental para entender el concepto de psicopatía del CAPP, es la dimensión central, la que mejor correlaciona con todas las demás en ambos grupos. Emocional y Self correlacionan bien en varones con todas las dimensiones, sin embargo, en mujeres, sus correlaciones con Conductual y Cognitivo pierden potencia.

Tabla 2. Matriz de correlaciones del PCL-R (total, factores y facetas) y del CAPP (total y dominios) para la muestra total, para mujeres (negrita) y para varones (cursiva).

\begin{tabular}{|c|c|c|c|c|c|c|c|c|c|c|c|c|c|c|}
\hline & $\begin{array}{l}\text { PCLR } \\
\text { total }\end{array}$ & PCLR F1 & PCLR F2 & PCLR f1 & PCLR f 2 & PCLR $\mathrm{f}_{3}$ & PCLR f 4 & CAPP A & CAPP Con & CAPP Cog & CAPP D & CAPPE & CAPP S & $\overline{\text { CAPP total }}$ \\
\hline $\begin{array}{l}\text { PCLR } \\
\text { total }\end{array}$ & 1,00 & & & & & & & & & & & & & \\
\hline PCLR F1 & $\begin{array}{l}0,83 \\
\mathbf{0 , 6 9} \\
0,85\end{array}$ & 1,00 & & & & & & & & & & & & \\
\hline PCLR F2 & $\begin{array}{l}0,86 \\
\mathbf{0 , 7 8} \\
0,88\end{array}$ & $\begin{array}{l}0,46 \\
\mathbf{0 , 1 1} \\
0,53\end{array}$ & 1,00 & & & & & & & & & & & \\
\hline PCLR f1 & $\begin{array}{l}0,76 \\
\mathbf{0 , 7 0} \\
0,77\end{array}$ & $\begin{array}{l}0,90 \\
\mathbf{0 , 9 4} \\
0,89\end{array}$ & $\begin{array}{l}0,43 \\
\mathbf{0 , 1 7} \\
0,49\end{array}$ & 1,00 & & & & & & & & & & \\
\hline PCLR f2 & $\begin{array}{l}0,73 \\
\mathbf{0 , 5 8} \\
0,74\end{array}$ & $\begin{array}{l}0,89 \\
\mathbf{0 , 9 3} \\
0,89\end{array}$ & $\begin{array}{l}0,39 \\
\mathbf{0 , 0 2} \\
0,55\end{array}$ & $\begin{array}{l}0,59 \\
\mathbf{0 , 7 5} \\
0,58\end{array}$ & 1,00 & & & & & & & & & \\
\hline PCLR f3 & $\begin{array}{l}0,83 \\
\mathbf{0 , 8 4} \\
0,83\end{array}$ & $\begin{array}{l}0,47 \\
\mathbf{0 , 2 6} \\
0,51\end{array}$ & $\begin{array}{l}0,93 \\
\mathbf{0 , 9 5} \\
0,93\end{array}$ & $\begin{array}{l}0,47 \\
\mathbf{0 , 3 2} \\
0,50\end{array}$ & $\begin{array}{l}0,37 \\
\mathbf{0 , 1 6} \\
0,40\end{array}$ & 1,00 & & & & & & & & \\
\hline PCLR f4 & $\begin{array}{l}0,72 \\
\mathbf{0 , 5 0} \\
0,76\end{array}$ & $\begin{array}{l}0,36 \\
\underline{\mathbf{0 , 1 7}} \\
0,44\end{array}$ & $\begin{array}{l}0,88 \\
\mathbf{0 , 8 6} \\
0,89\end{array}$ & $\begin{array}{l}0,29 \\
\mathbf{0 , 1 2} \\
0,37\end{array}$ & $\begin{array}{l}0,35 \\
\underline{\mathbf{0 , 2 0}} \\
0,42\end{array}$ & $\begin{array}{l}0,64 \\
\mathbf{0 , 6 6} \\
0,65\end{array}$ & 1,00 & & & & & & & \\
\hline CAPP A & $\begin{array}{l}0,60 \\
\mathbf{0 , 5 7} \\
0,60\end{array}$ & $\begin{array}{l}0,64 \\
\mathbf{0 , 6 5} \\
0,64\end{array}$ & $\begin{array}{l}0,41 \\
\mathbf{0 , 2 7} \\
0,44\end{array}$ & $\begin{array}{l}0,45 \\
\mathbf{0 , 4 8} \\
0,44\end{array}$ & $\begin{array}{l}0,70 \\
\mathbf{0 , 7 5} \\
0,69\end{array}$ & $\begin{array}{l}0,33 \\
\mathbf{0 , 2 6} \\
0,34\end{array}$ & $\begin{array}{l}0,43 \\
\mathbf{0 , 2 2} \\
0,47\end{array}$ & 1,00 & & & & & & \\
\hline CAPP Con & $\begin{array}{l}0,62 \\
\mathbf{0 , 5 1} \\
0,63\end{array}$ & $\begin{array}{l}0,41 \\
\mathbf{0 , 0 5} \\
0,45\end{array}$ & $\begin{array}{l}0,65 \\
\mathbf{0 , 6 8} \\
0,66\end{array}$ & $\begin{array}{l}0,37 \\
\mathbf{0 , 0 4} \\
0,41\end{array}$ & $\begin{array}{l}0,36 \\
\mathbf{0 , 0 5} \\
0,40\end{array}$ & $\begin{array}{l}0,60 \\
\mathbf{0 , 6 3} \\
0,60\end{array}$ & $\begin{array}{l}0,57 \\
\mathbf{0 , 6 0} \\
0,59\end{array}$ & $\begin{array}{l}0,55 \\
\mathbf{0 , 3 2} \\
0,57\end{array}$ & 1,00 & & & & & \\
\hline $\begin{array}{l}\text { CAPP } \\
\text { Cog }\end{array}$ & $\begin{array}{l}0,58 \\
\mathbf{0 , 3 9} \\
0,61\end{array}$ & $\begin{array}{l}0,49 \\
\mathbf{0 , 2 2} \\
0,51\end{array}$ & $\begin{array}{l}0,52 \\
\mathbf{0 , 4 1} \\
0,55\end{array}$ & $\begin{array}{l}0,37 \\
\mathbf{0 , 2 1} \\
0,39\end{array}$ & $\begin{array}{l}0,50 \\
\mathbf{0 , 2 1} \\
0,53\end{array}$ & $\begin{array}{l}0,46 \\
\mathbf{0 , 4 1} \\
0,48\end{array}$ & $\begin{array}{l}0,48 \\
\mathbf{0 , 3 2} \\
0,52\end{array}$ & $\begin{array}{l}0,70 \\
\mathbf{0 , 2 9} \\
0,74\end{array}$ & $\begin{array}{l}0,75 \\
\mathbf{0 , 7 0} \\
0,75\end{array}$ & 1,00 & & & & \\
\hline CAPP D & $\begin{array}{l}0,68 \\
\mathbf{0 , 3 3} \\
0,72\end{array}$ & $\begin{array}{l}0,72 \\
\mathbf{0 , 6 4} \\
0,74\end{array}$ & $\begin{array}{l}0,46 \\
\underline{\mathbf{0 , 0 3}} \\
0,54\end{array}$ & $\begin{array}{l}0,62 \\
0,57 \\
0,62\end{array}$ & $\begin{array}{l}0,67 \\
\mathbf{0 , 6 3} \\
0,68\end{array}$ & $\begin{array}{c}0,41 \\
\mathbf{0 , 0 0 4} \\
0,47\end{array}$ & $\begin{array}{l}0,42 \\
\underline{\mathbf{0 , 1 4}} \\
0,51\end{array}$ & $\begin{array}{l}0,80 \\
\mathbf{0 , 5 6} \\
0,82\end{array}$ & $\begin{array}{l}0,63 \\
\mathbf{0 , 3 0} \\
0,65\end{array}$ & $\begin{array}{l}0,78 \\
\mathbf{0 , 3 3} \\
0,81\end{array}$ & 1,00 & & & \\
\hline CAPP E & $\begin{array}{l}0,64 \\
\mathbf{0 , 2 6} \\
0,68\end{array}$ & $\begin{array}{l}0,61 \\
\mathbf{0 , 4 3} \\
0,63\end{array}$ & $\begin{array}{l}0,50 \\
\mathbf{0 , 0 5} \\
0,57\end{array}$ & $\begin{array}{l}0,43 \\
\mathbf{0 , 3 2} \\
0,44\end{array}$ & $\begin{array}{l}0,65 \\
\mathbf{0 , 4 9} \\
0,67\end{array}$ & $\begin{array}{l}0,43 \\
\mathbf{0 , 1 1} \\
0,49\end{array}$ & $\begin{array}{l}0,48 \\
\underline{\mathbf{0 , 0 7}} \\
0,55\end{array}$ & $\begin{array}{l}0,79 \\
\mathbf{0 , 5 2} \\
0,82\end{array}$ & $\begin{array}{l}0,61 \\
\mathbf{0 , 3 6} \\
0,63\end{array}$ & $\begin{array}{l}0,78 \\
\mathbf{0 , 2 5} \\
0,80\end{array}$ & $\begin{array}{l}0,91 \\
\mathbf{0 , 7 5} \\
0,92\end{array}$ & 1,00 & & \\
\hline CAPP S & $\begin{array}{l}0,62 \\
\mathbf{0 , 4 8} \\
0,65\end{array}$ & $\begin{array}{l}0,67 \\
\mathbf{0 , 7 0} \\
0,67\end{array}$ & $\begin{array}{l}0,41 \\
\mathbf{0 , 1 3} \\
0,48\end{array}$ & $\begin{array}{l}0,64 \\
\mathbf{0 , 6 6} \\
0,64\end{array}$ & $\begin{array}{l}0,55 \\
\mathbf{0 , 6 6} \\
0,55\end{array}$ & $\begin{array}{l}0,37 \\
\mathbf{0 , 2 0} \\
0,41\end{array}$ & $\begin{array}{l}0,37 \\
\underline{\mathbf{0 , 0 1}} \\
0,47\end{array}$ & $\begin{array}{l}0,68 \\
\mathbf{0 , 6 0} \\
0,70\end{array}$ & $\begin{array}{l}0,59 \\
\mathbf{0 , 2 1} \\
0,63\end{array}$ & $\begin{array}{l}0,67 \\
\mathbf{0 , 2 3} \\
0,70\end{array}$ & $\begin{array}{l}0,84 \\
\mathbf{0 , 7 2} \\
0,85\end{array}$ & $\begin{array}{l}0,73 \\
\mathbf{0 , 4 3} \\
0,75\end{array}$ & 1,00 & \\
\hline $\begin{array}{l}\text { CAPP } \\
\text { total }\end{array}$ & $\begin{array}{l}0,71 \\
\mathbf{0 , 5 9} \\
0,74\end{array}$ & $\begin{array}{l}0,69 \\
\mathbf{0 , 6 4} \\
0,70\end{array}$ & $\begin{array}{l}0,55 \\
\mathbf{0 , 3 3} \\
0,61\end{array}$ & $\begin{array}{l}0,57 \\
\mathbf{0 , 5 5} \\
0,58\end{array}$ & $\begin{array}{l}0,65 \\
\mathbf{0 , 6 5} \\
0,66\end{array}$ & $\begin{array}{l}0,49 \\
\mathbf{0 , 3 7} \\
0,53\end{array}$ & $\begin{array}{l}0,51 \\
\mathbf{0 , 2 0} \\
0,58\end{array}$ & $\begin{array}{l}0,84 \\
\mathbf{0 , 7 4} \\
0,86\end{array}$ & $\begin{array}{l}0,78 \\
\mathbf{0 , 6 4} \\
0,80\end{array}$ & $\begin{array}{l}0,87 \\
\mathbf{0 , 6 1} \\
0,89\end{array}$ & $\begin{array}{l}0,96 \\
\mathbf{0 , 8 6} \\
0,96\end{array}$ & $\begin{array}{l}0,91 \\
\mathbf{0 , 7 2} \\
0,92\end{array}$ & $\begin{array}{l}0,88 \\
\mathbf{0 , 7 8} \\
0,89\end{array}$ & 1,00 \\
\hline
\end{tabular}

Nota. PCLR: Psychopathy Checklist Revised; PCLR: F1: factor 1; F2: factor 2; f1: faceta 1 (interpersonal); f2: faceta 2 (afectivo); f3: faceta 3 (estilo de vida); f4: faceta 4 (conducta antisocial); CAPP: Comprehensive Assessment of Psychopathic Personality CAPP: A: apego; Con: conductual; Cog: cognitivo; D: dominancia; E: emocional; S: Self. Subrayado: puntuación negativa.

La Tabla 3 indica que a nivel del análisis univariante las diferencias significativas entre hombres y mujeres para el CAPP se encuentran fundamentalmente en las dimensiones de Dominancia (puntuación total, e ítems 23 y 30), Emocional (ítems 5, 31 y 16), y especialmente Self (pun- tuación total, e ítems 20,14, 1, 22, 2 y 21). También se encontró una diferencia significativa en el ítem 8 de Apego. Todas estas diferencias significativas indican puntuaciones más elevadas para las mujeres. 
Psicopatía y adicciones en mujeres: Estudio comparativo utilizando el Psychopathy Checklist Revised y el Comprehensive Assessment of Psychopathic Personality

Tabla 3. Comparación univariante entre mujeres y varones para los ítems del CAPP, incluyendo sus dominios y puntuación total.

\begin{tabular}{|c|c|c|c|c|}
\hline & $\begin{array}{l}\text { Mujeres media } \\
\text { (DS) }\end{array}$ & $\begin{array}{c}\text { Varones media } \\
\text { (DS) }\end{array}$ & $\mathbf{F}$ & $\mathbf{P}$ \\
\hline Apego 18 desapegado & $2,89(1,61)$ & $2,61(1,77)$ & 0,61 & 0,43 \\
\hline Apego 8 no adquiere compromisos & $3,14(1,55)$ & $2,56(1,59)$ & 3,17 & $<0,01$ \\
\hline Apego 25 sin empatía & $2,03(1,66)$ & $2,00(1,72)$ & 0,01 & 0,93 \\
\hline Apego 24 insolidario & $2,17(1,56)$ & $2,26(1,66)$ & 0,06 & 0,80 \\
\hline Conductual 3 inconstante & $1,75(1,64)$ & $1,88(1,85)$ & 0,12 & 0,72 \\
\hline Conductual 26 no fiable & $2,57(1,55)$ & $2,32(1,87)$ & 0,44 & 0,50 \\
\hline Conductual 15 imprudente & $2,71(1,58)$ & $2,51(1,70)$ & 0,35 & 0,55 \\
\hline Conductual 6 intranquilo & $2,60(1,39)$ & $2,02(1,75)$ & 2,75 & 0,09 \\
\hline Conductual 17 disruptivo & $2,32(1,44)$ & $1,87(1,65)$ & 1,82 & 0,17 \\
\hline Conductual 32 agresivo & $1,57(1,20)$ & $1,61(1,59)$ & 0,02 & 0,89 \\
\hline Cognitivo 19 suspicaz & $3,60(1,59)$ & $3,11(1,77)$ & 1,92 & 0,16 \\
\hline Cognitivo 28 falta de capacidad de concentración & $1,75(1,71)$ & $1,34(1,60)$ & 1,51 & 0,22 \\
\hline Cognitivo 7 intolerante & $1,85(1,38)$ & $1,40(1,55)$ & 2,13 & 0,14 \\
\hline Cognitivo 27 inflexible & $2,17(1,67)$ & $1,97(1,51)$ & 0,44 & 0,51 \\
\hline Cognitivo 29 ausencia de planificación & $2,57(1,79)$ & $2,07(1,92)$ & 1,64 & 0,20 \\
\hline Dominancia 11 oposicionista & $1,32(1,15)$ & $1,26(1,44)$ & 0,04 & 0,83 \\
\hline Dominancia 12 dominante & $2,14(1,75)$ & $1,69(1,63)$ & 1,75 & 0,18 \\
\hline Dominancia 10 falso & $2,89(1,52)$ & $2,44(1,71)$ & 1,72 & 0,19 \\
\hline Dominancia 9 manipulador & $3,14(1,64)$ & $2,55(1,63)$ & 3,16 & 0,07 \\
\hline Dominancia 23 no sincero & $3,35(1,33)$ & $2,68(1,67)$ & 4,15 & 0,04 \\
\hline Dominancia 30 charlatán & $2,71(1,65)$ & $2,01(1,58)$ & 4,69 & 0,03 \\
\hline Emocional 5 sin ansiedad & $3,42(1,39)$ & $2,67(1,63)$ & 5,3 & 0,02 \\
\hline Emocional 33 sin capacidad de experimentar placer & $1,39(1,06)$ & $1,59(1,61)$ & 0,42 & 0,51 \\
\hline Emocional 4 sin profundidad emocional & $1,53(1,29)$ & $1,86(1,72)$ & 0,92 & 0,33 \\
\hline Emocional 31 sin estabilidad emocional & $3,10(1,25)$ & $1,90(1,74)$ & 12,22 & $<0,01$ \\
\hline Emocional 16 sin remordimientos & $4,17(1,27)$ & $3,31(1,69)$ & 6,68 & 0,01 \\
\hline Self 20 centrado en sí mismo & $3,64(1,63)$ & $2,76(1,99)$ & 4,85 & 0,02 \\
\hline Self 14 exaltación de sí mismo & $2,53(1,77)$ & $1,79(1,73)$ & 4,36 & 0,03 \\
\hline Self 1 se siente único & $3,03(1,79)$ & $2,22(1,84)$ & 4,72 & 0,03 \\
\hline Self 13 se siente con excesivos derechos & $2,67(1,72)$ & $2,02(1,66)$ & 3,64 & 0,05 \\
\hline Self 22 se siente invulnerable & $3,10(1,66)$ & $1,78(1,67)$ & 15,08 & $<0,01$ \\
\hline Self 2 autojustificación & $3,89(1,47)$ & $3,29(1,64)$ & 3,29 & 0,07 \\
\hline Self 21 concepto de sí mismo inestable & $2,00(1,74)$ & $1,18(1,60)$ & 6,02 & 0,01 \\
\hline CAPP Apego & $10,25(5,22)$ & $9,44(5,87)$ & 0,46 & 0,49 \\
\hline CAPP Conductual & $13,54(6,75)$ & $12,23(8,50)$ & 0,6 & 0,44 \\
\hline CAPP Cognitivo & $11,96(4,19)$ & $9,90(5,99)$ & 3,05 & 0,08 \\
\hline CAPP Dominancia & $29,21(8,56)$ & $24,00(13,07)$ & 4,15 & 0,04 \\
\hline CAPP Emocional & $13,64(2,88)$ & $11,35(6,22)$ & 3,64 & 0,05 \\
\hline CAPP Self & $20,89(7,21)$ & $15,08(9,26)$ & 10,03 & $<0,01$ \\
\hline CAPP total & $99,50(25,80)$ & $82,03(43,62)$ & 4,24 & 0,04 \\
\hline
\end{tabular}

Nota. CAPP: Comprehensive Assessment of Psychopathic Personality. Negrilla: resultados estadísticamente significativos; DS: Desviación estándar.

La Tabla 4 indica que a nivel del análisis univariante las diferencias significativas entre hombres y mujeres para el PCL-R se encuentran fundamentalmente con la impulsividad (ítems 10 y 11) y la conducta antisocial (faceta 4), en este caso las puntuaciones son significativamente más elevadas para los varones. Las mujeres obtuvieron puntuaciones significativamente más elevadas para un ítem, el 17, que mide las relaciones maritales de corta duración. 
Tabla 4. Comparación univariante entre mujeres y varones para los ítems del PCL-R, incluyendo sus factores, facetas y puntuación total.

\begin{tabular}{|c|c|c|c|c|}
\hline & Mujeres media (DS) & Varones media (DS) & $\mathbf{F}$ & $\mathbf{P}$ \\
\hline PCL-R 1 facilidad de palabra / encanto superficial & $1,00(0,98)$ & $0,71(0,86)$ & 2,59 & 0,10 \\
\hline PCL-R 2 sentido desmesurado de auto valía & $0,92(0,90)$ & $0,73(0,90)$ & 1,06 & 0,30 \\
\hline PCL-R 3 necesidad de estimulación / tendencia al aburrimiento & $1,21(0,95)$ & $1,31(0,92)$ & 0,30 & 0,58 \\
\hline PCL-R 4 mentirosos patológico & $0,92(0,94)$ & $0,86(0,94)$ & 0,10 & 0,75 \\
\hline PCL-R 5 estafador y manipulador & $1,53(0,69)$ & $1,47(0,83)$ & 0,15 & 0,70 \\
\hline PCL-R 6 ausencia de remordimiento o sentimiento de culpa & $1,67(0,72)$ & $1,57(0,75)$ & 0,47 & 0,49 \\
\hline PCL-R 7 afecto superficial y poco profundo & $0,60(0,83)$ & $0,72(0,89)$ & 0,45 & 0,50 \\
\hline PCL-R 8 insensibilidad afectiva y ausencia de empatía & $0,75(0,88)$ & $0,86(0,89)$ & 0,39 & 0,53 \\
\hline PCL-R 9 estilo de vida parasitario & $1,21(0,95)$ & $0,97(0,94)$ & 1,50 & 0,22 \\
\hline PCL-R 10 pobre auto control de la conducta & $1,28(0,89)$ & $1,66(0,68)$ & 6,68 & 0,01 \\
\hline PCL-R 11 conducta sexual promiscua & $0,92(1,01)$ & $0,73(0,94)$ & 1,01 & 0,31 \\
\hline PCL-R 12 problemas de conducta en la infancia & $0,60(0,91)$ & $0,64(0,91)$ & 0,04 & 0,85 \\
\hline PCL-R 13 ausencia de metas realistas a largo plazo & $0,96(0,99)$ & $0,92(0,97)$ & 0,04 & 0,84 \\
\hline PCL-R 14 impulsividad & $1,32(0,83)$ & $1,68(0,65)$ & 6,58 & 0,01 \\
\hline PCL-R 15 irresponsabilidad & $1,60(0,73)$ & $1,72(0,64)$ & 0,73 & 0,39 \\
\hline PCL-R 16 incapacidad de aceptar la responsabilidad de las propias acciones & $1,82(0,47)$ & $1,58(0,75)$ & 2,60 & 0,10 \\
\hline PCL-R 17 frecuentes relaciones maritales de corta duración & $0,53(0,88)$ & $0,22(0,56)$ & 6,23 & 0,01 \\
\hline PCL-R 18 delincuencia juvenil & $0,10(0,41)$ & $0,37(0,75)$ & 3,37 & 0,06 \\
\hline PCL-R 19 revocación de la libertad condicional & $0,28(0,71)$ & $0,54(0,87)$ & 2,23 & 0,13 \\
\hline PCL-R 20 versatilidad criminal & $0,10(0,41)$ & $0,32(0,71)$ & 2,46 & 0,11 \\
\hline factor 1 & $9,25(4,55)$ & $8,54(4,65)$ & 0,57 & 0,45 \\
\hline factor 2 & $8,71(5,24)$ & $10,17(5,05)$ & 2 & 0,15 \\
\hline faceta 1 interpersonal & $4,39(2,60)$ & $3,79(2,64)$ & 1,26 & 0,26 \\
\hline faceta 2 afectivo & $4,85(2,27)$ & $4,75(2,59)$ & 0,04 & 0,83 \\
\hline faceta 3 estilo de vida & $6,32(3,58)$ & $6,62(3,08)$ & 0,22 & 0,63 \\
\hline faceta 4 conducta antisocial & $2,39(2,14)$ & $3,55(2,46)$ & 5,51 & 0,02 \\
\hline Total & $19,32(8,38)$ & $19,60(9,07)$ & 0,02 & 0,87 \\
\hline
\end{tabular}

Nota. DS: Desviación estándar; PCL-R: Psychopathy Checklist Revised. Negrilla: resultados estadísticamente significativos.

En conjunto, se observa a nivel del análisis univariante, una mayor capacidad del CAPP frente al PCL-R para distinguir entre mujeres y varones. Ya se puede apreciar este hecho en las puntuaciones totales, para el CAPP hay una diferencia significativa a favor de las mujeres que no existe en el PCL-R.

Once $(39,28 \%)$ mujeres puntuaron en el PCL-R 25 o más superando el punto de corte europeo frente a 69 $(39,20 \%)$ varones. La comparación univariante en este grupo de internos arrojó las siguientes diferencias significativas a favor de los varones, con la excepción del ítem 17: pobre autocontrol de la conducta (ítem 10) $(\mathrm{W}=436,5, \mathrm{p}=$ $0,036)$, irresponsabilidad (ítem 14) $(\mathrm{W}=465, \mathrm{p}<0,01)$, frecuentes relaciones maritales de corta duración (ítem 17) ( $\mathrm{W}=226.5, \mathrm{p}=0,01)$, delincuencia juvenil (ítem 18) (W $=507,5, \mathrm{p}=0,037)$, revocación de la libertad condicional (ítem 19) $(\mathrm{W}=508,5, \mathrm{p}=0,03)$, factor $2(\mathrm{~W}=541, \mathrm{p}=0,02)$, faceta $4(\mathrm{~W}=554, \mathrm{p}=0,01)$; y para el CAPP: sin profundidad emocional (emocional 4) $(\mathrm{W}=549, \mathrm{p}=0,01)$, en este caso más elevado para las mujeres.
Los resultados de la regresión logística se presentan en la Tabla 5, en dicha tabla se puede apreciar que únicamente resultan estadísticamente significativas las variables PCL-R faceta 4 y CAPP Self, mientras que las variables CAPP Dominancia y CAPP Emocional presentan un valor p por debajo de 0,25. El Test de Homer y Lemeshow, test de bondad de ajuste del modelo propuesto arroja un resultado según el cual el modelo obtenido se ajusta a la realidad.

La Tabla 6 presenta los resultados obtenidos de la repetición del modelo de regresión logística utilizando únicamente como variables del mismo PCL-R Faceta 4, CAPP Dominancia, CAPP Emocional y CAPP Self. Es decir, aquellas variables que en el modelo original presentaban un valor $\mathrm{p}$ por debajo de 0,25. A partir de los resultados obtenidos, se observa claramente cómo continúan siendo significativas únicamente las dos variables identificadas como tales en el primer modelo. En este caso, el resultado obtenido por el Test de Homer y Lemeshow también permite afirmar que el modelo se ajusta a la realidad. 
Tabla 5. Regresión logística cuya variable dependiente es el sexo del individuo, utilizando las facetas del PCL-R y los dominios del CAPP.

\begin{tabular}{|c|c|c|c|c|c|}
\hline Variables & B & ES & OR (IC 95\%) & gl & $\mathbf{p}$ \\
\hline PCLR-Faceta 1 & $-0,01$ & 0,15 & $0,99(0,74 ; 1,34)$ & 1 & 0,98 \\
\hline PCLR-Faceta 2 & 0,11 & 0,15 & $1,12(0,82 ; 1,52)$ & 1 & 0,45 \\
\hline PCLR-Faceta 3 & $-0,03$ & 0,11 & $0,96(0,77 ; 1,19)$ & 1 & 0,72 \\
\hline PCLR-Faceta 4 & 0,53 & 0,16 & $1,69(1,22 ; 2,35)$ & 1 & $<0,01$ \\
\hline CAPP-Apego & 0,06 & 0,07 & $1,06(0,92 ; 1,22)$ & 1 & 0,35 \\
\hline CAPP-Conductual & $-0,02$ & 0,04 & $0,97(0,88 ; 1,07)$ & 1 & 0,61 \\
\hline CAPP-Cognitivo & $-0,05$ & 0,07 & $0,94(0,81 ; 1,09)$ & 1 & 0,42 \\
\hline CAPP-Dominancia & 0,07 & 0,06 & $1,08(0,94 ; 1,23)$ & 1 & 0,24 \\
\hline CAPP-Emocional & $-0,10$ & 0,08 & $0,90(0,75 ; 1,07)$ & 1 & 0,24 \\
\hline CAPP-Self & $-0,12$ & 0,04 & $0,87(0,79 ; 0,96)$ & 1 & $<0,01$ \\
\hline
\end{tabular}

Nota. PCLR: Psychopathy Checklist Revised; CAPP: Comprehensive Assessment of Psychopathic Personality; ES: Error estándar; OR: Odds Ratio; IC: Intervalo de Confianza; gl: grados de libertad.

Tabla 6. Modelo de regresión logística incluyendo las variables de PCL-R y CAPP que en el modelo global obtuvieron un $p$-valor por debajo de o,2.

\begin{tabular}{lccccc} 
Variables & B & ES & OR $(\mathbf{I C} \mathbf{9 5} \%)$ & gl & p-valor \\
\hline PCLR-Faceta 4 & 0,42 & 0,12 & $1,52(1,20 ; 1,93)$ & 1 & $<\mathbf{0 , 0 1}$ \\
\hline $\begin{array}{l}\text { CAPP- } \\
\text { Dominancia }\end{array}$ & 0,08 & 0,05 & $1,09(0,97 ; 1,22)$ & 1 & 0,11 \\
\hline $\begin{array}{l}\text { CAPP- } \\
\text { Emocional }\end{array}$ & $-0,08$ & 0,06 & $0,91(0,80 ; 1,04)$ & 1 & 0,18 \\
\hline CAPP-Self & $-0,12$ & 0,04 & $0,87(0,80 ; 0,95)$ & 1 & $<\mathbf{0 , 0 1}$ \\
\hline
\end{tabular}

Nota. PCLR: Psychopathy Checklist Revised; CAPP: Comprehensive Assessment of Psychopathic Personality; ES: Error estándar; OR: Odds Ratio; IC: Intervalo de Confianza; gl: grados de libertad.

A continuación, y con el fin de conocer si resulta posible diferenciar entre hombres y mujeres en función de los resultados obtenidos en los test CAPP y PCL-R, se aplicaron modelos basados en Máquinas de Vectores Soporte. En primer lugar, se realizó un modelo que utiliza como información para su entrenamiento los datos correspondientes a todos los individuos disponibles, empleando como variables de entrada todas las variables de CAPP y PCL-R, y como variable de salida el sexo. El modelo obtuvo unos valores de especificidad y sensibilidad del $100 \%$.

Sin embargo, este resultado, no indica que el modelo de Máquinas de Vectores Soporte tenga capacidad predictiva alguna, sino simplemente que es capaz de clasificar de forma correcta todo el conjunto de datos, una vez conocida el $100 \%$ de la información. Con el fin de conocer la capacidad predictiva del modelo, y por tanto saber si a partir de los resultados en las pruebas CAPP y PCL-R es posible discriminar entre hombres y mujeres, el conjunto de datos se divide de manera aleatoria en muestras de entrenamiento y validación. Cada una de las muestras de entrenamiento está formada por el $66,66 \%$ de la información disponible, y las de validación por el 33,34\% restante. Así, del total de
204 individuos a los que se les realizó tanto el test CAPP como el PCL-R, aleatoriamente se eligen para cada conjunto de entrenamiento 117 hombres y 20 mujeres. Para cada uno de los kernels disponibles, se repitió el proceso de entrenamiento y validación 10.000 veces. En el caso del kernel lineal, el valor medio de sensibilidad obtenido fue del $0 \%$ y el de especificidad del $50 \%$, para el kernel polinómico de grado 7 (el mejor de todos los grados), el valor de sensibilidad fue del 22,22\% y el de especificidad del $35,59 \%$, para el kernel sigmoidal el valor de sensibilidad fue del $0 \%$ y el de especificidad del $47,46 \%$ mientras que, finalmente, para el radial, el valor medio de sensibilidad fue del $0 \%$ y el de especificidad del $50 \%$. La matriz de confusión para el kernel polinómico de grado 7 indicaba los siguieintes grados de predicción real: Varones - Varones [44,45 (44,37 - 44,52)], Varones - Mujeres [6,35 (6,32 $6,38)]$, Mujeres - Varones $[14,55(14,47 ; 14,62)]$ y Mujeres - Mujeres [2,64 (2,61; 2,67)].

Estos resultados indican que, en esta muestra, al modelo de Máquina de Vectores Soporte le resulta muy complicado a partir de las variables de CAPP y PCL-R distinguir a las mujeres, grupo minoritario, de los hombres.

\section{Discusión}

Las variables sociales y demográficas analizadas indican que ambos grupos, mujeres y varones, no presentan diferencias significativas que pudiesen funcionar como variables de confusión para el análisis posterior. Las diferencias con respecto al consumo de tóxicos están equilibradas. El alcohol y el cánnabis, más consumidos por los varones, son consumidos de forma muy frecuente en toda la muestra. En esta muestra, el alcohol se relaciona poco con las conductas antisociales y la psicopatía, justo lo contrario que el cánnabis. Además, no hay diferencias significativas con respecto a las sustancias que más se relacionan con la con- 
ducta criminal, los derivados mórficos y los estimulantes como la cocaína y las anfetaminas. Finalmente, la presencia de TP indica ya una diferencia entre mujeres y varones ya descrita en estudios previos (Carabellese et al., 2019; Dolan y Vollm, 2009; Gray y Snowden, 2016; Guay et al., 2018; Nicholls et al., 2005; Thomson, 2017; Verona et al., 2013; Wynn et al., 2012). Los varones, presentan una incidencia superior de TAP, el TP que más se relaciona con la conducta criminal, mientras que las mujeres presentaban una incidencia superior de trastornos que se relacionan con la ausencia de empatía y el estilo interpersonal arrogante y grandioso, es decir narcisista, borderline e histriónico. El TP dependiente, también más prevalente en mujeres, presenta una baja frecuencia en la muestra. Es necesario recordar que, pese a estas diferencias significativas en lo referente a las prevalencias de trastornos de la personalidad, no las hay en lo referente a la comisión de delitos.

La matriz de correlaciones muestra una evolución de las puntuaciones del PCL-R y del CAPP muy similar para ambos grupos. Las correlaciones entre factores y facetas del PCL-R y las dimensiones del CAPP presentan una distribución y agrupación muy similar. En las mujeres destaca la elevada correlación entre la faceta 2 del PCL-R y los dominios de Apego y Self del CAPP. Por otro lado, en los varones destaca una mejor correlación de las dimensiones del CAPP Conductual y Cognitivo con el resto de dimensiones del mismo instrumento.

El análisis multivariante profundiza en los hallazgos previos ya que demuestra que el CAPP, especialmente las dimensiones Emocional, Dominancia y Self, permite diferenciar a varones de mujeres, obteniendo estas últimas puntuaciones significativamente más elevadas. El PCL-R, por su parte, permite discriminar entre géneros a través, fundamentalmente, de la faceta 4 , la conducta antisocial, significativamente más elevada en varones, al igual que en estudios previos (Carabellese et al., 2019; Dolan y Vollm, 2009; Gray y Snowden, 2016; Guay et al., 2018; Nicholls et al., 2005; Thomson, 2017; Verona et al., 2013; Wynn et al., 2012) . Los resultados del PCL-R concuerdan con la investigación previa, pero los del CAPP, más novedosos, nos indican que la valoración de la psicopatía en mujeres estaría infravalorada si el PCL-R no se completa con este otro instrumento, más sensible a la hora de valorar la psicopatología de la personalidad. El hecho de que no haya diferencias significativas en la prevalencia de comisión de delitos violentos, ni en el porcentaje de varones y mujeres que superan el punto de corte europeo para la psicopatía en el PCL-R (puntuación de 25 o más) refuerzan la importancia de estos hallazgos.

El análisis univariante de los internos con puntuación en el PCL-R de 25 o más arroja otro dato de gran interés. La faceta 4 sigue marcando una diferencia significativa entre mujeres y varones, con puntuaciones más elevadas para estos últimos. Esta diferencia indica la importancia de esta faceta en las puntuaciones del PCL-R en la muestra masculina. Los varones precisan de elevadas puntaciones en esta faceta para entrar en el rango de la psicopatía, sin embargo, las mujeres, en comparación con los varones, alcanzan las puntuaciones de rango psicopático de una forma más balanceada entre facetas. Es decir, los hombres para ser considerados psicopáticos desde el punto de vista del PCL-R, suelen tener que ser altamente antisociales, las mujeres no. Desafortunadamente, el punto de corte del PCL-R no es extrapolable al CAPP, y el CAPP no dispone de puntos de corte propios. Por ello no podemos afirmar con rotundidad que las dimensiones del CAPP no presentan diferencias significativas entre mujeres y varones más psicopáticos, sino que, simplemente, no las presentan cuando se utiliza un punto de corte de 25 en el PCL-R. Es fundamental en los TP de naturaleza dimensional y no categorial, no confundir un punto de corte con un diagnóstico.

La regresión logística, confirma que la faceta 4 del PCL-R en los varones, y el dominio Self del CAPP en las mujeres, son las variables, a nivel de facetas y dominios, más discriminatorias entre géneros. De nuevo se puede apreciar la importancia de combinar la valoración del PCL-R con la del CAPP.

¿Cómo interpretar la incapacidad del Modelo de Maquinas de Vectores Soporte para discriminar entre varones y mujeres? Una posibilidad podría ser que la muestra de mujeres fuera insuficiente, otra alternativa es que las variables significativas indicadas hasta ahora no tuviesen suficiente potencia para superar este último nivel de exigencia. Esta última hipótesis indicaría la imposibilidad de crear un modelo predictivo para diferenciar a las mujeres de los varones utilizando el PCL-R junto al CAPP para valorar la psicopatía. Es decir, que no podríamos indicar al personal clínico y forense que la faceta 4 del PCL-R, a partir de una determinada puntuación, permite detectar a los varones de una muestra, y a la inversa, para las mujeres, el dominio Self del CAPP. Esto no invalida los resultados previos obtenidos en el análisis univariante y en la regresión logística. No es de extrañar que no haya una variable en el CAPP o en el PCL-R que permita, en base a su puntuación, diferenciar a las mujeres de los varones, después de todo la investigación previa no ha establecido que haya un síntoma, signo, conducta o delito que no pueda estar presente en su máxima intensidad tanto en mujeres como en varones.

Este estudio presenta limitaciones. Ya se ha indicado previamente que en esta muestra el número de mujeres es representativo pero pequeño. Para alcanzar un mayor poder estadístico sería necesario aumentar el número de mujeres en la muestra. Esto plantea un problema, para que la muestra no sea de conveniencia y siga siendo representativa de la población penitenciaria española, el número de varones incluidos en el estudio también debe incrementarse proporcionalmente, complicando y encareciendo el estudio. Por otro lado, aunque el objetivo de los investi- 
gadores era mantener el ciego entre los resultados de los instrumentos de la valoración de la psicopatía y los TP del resto de las variables socio-demográficas, de consumo y de delitos, dado que el PCL-R, CAPP e IPDE exigen de la realización de una entrevista, es posible que alguno de los internos rompiese, de forma involuntaria, la situación de ciego durante dichas entrevistas.

En conclusión, en este estudio se demuestra que existen diferencias significativas entre mujeres y varones cuando se les aplican los mismos instrumentos para la valoración de la psicopatía y ambos grupos están balanceados para el consumo de sustancias. Las mujeres presentan más psicopatología de la personalidad que los varones, sobre todo en lo referente al self, y en menor medida a las alteraciones emocionales y la dominancia. Por ello en esta muestra la presencia de TP de tipo narcisista, borderline e histriónico son más frecuentes en mujeres. Es fundamental recordar que es el CAPP el instrumento que capta esta diferencia con claridad, no el PCL-R. Por el contrario, los varones en comparación con las mujeres presentan una mayor prevalencia de conductas antisociales, y por ello se les diagnostica con más frecuencia de TAP. De nuevo, es fundamental indicar que esta diferencia se detecta con el PCL-R y no con el CAPP. Por todo esto, es necesario recordar al personal clínico y forense que para captar con claridad estas diferencias de género a la hora de valorar la psicopatía deben combinar los instrumentos, ya que si sólo utilizan el PCL-R perderán capacidad para captar parte de las diferencias psicopatológicas que hay entre mujeres y varones. Pese a estas diferencias, el constructo de la psicopatía es muy similar en ambos géneros, no existiendo ningún síntoma o ítem que discrimine con claridad predictiva a las mujeres de los varones.

\section{Agradecimientos}

Este trabajo está apoyado parcialmente por el Centro de Investigación Biomédica en Red de Salud Mental (CIBERSAM) y el Ministerio de Economía, Industria y Competitividad y por el Gobierno del Principado de Asturias PCTI 2018-2022 IDI/2018/235 con cofinanciación FEDER.

\section{Conflicto de intereses}

Los autores declaran que no existe ningún conflicto de intereses en relación a la realización de este estudio.

\section{Referencias}

Blackburn, R. (2007). Personality disorder and antisocial deviance: Comments on the debate on the structure of the psychopathy checklist-revised. Journal of Personality Disorders, 21, 142-159. doi:10.1521/pedi.2007.21.2.142.
Blair, R. J. y Lee, T. M. (2013). The social cognitive neuroscience of aggression, violence, and psychopathy. Social Neuroscience, 8, 108-111. doi:10.1080/17470919.2012.75 7869.

Cale, J., Lussier, P., McCuish, E. y Corrado, R. (2015). The prevalence of psychopathic personality disturbances among incarcerated youth: Comparing serious, chronic, violent and sex offenders. Journal of Criminal Justice, 43, 337-344. doi:10.1016/j.jcrimjus.2015.04.005.

Carabellese, F., Felthous, A. R., La Tegola, D., Rossetto, I., Montalbo, D., Franconi, F. y Catanesi, R. (2019). Psychopathy and female gender: Phenotypic expression and comorbidity; A study comparing a sample of women hospitalized in Italy's maximum security facility with women who were criminally sentenced and imprisoned. Journal of Forensic Sciences, 64, 1438-1443. doi:10.1111/15564029.14039.

Cooke, D. J., Hart, S. D., Logan, C. y Michie, C. (2012). Explicating the construct of psychopathy: Development and validation of a conceptual model, the Comprehensive Assessment of Psychopathic Personality (CAPP). International Journal of Forensic Mental Health, 11, 242-252. doi:10.1080/14999013.2012.746759.

Cooke, D. J. y Logan, C. (2015). Capturing clinical complexity: Towards a personality-oriented measure of psychopathy. Journal of Criminal Justice, 43, 262-273. doi:10.1016/j.jcrimjus.2015.04.004.

Cooke, D. J. y Michie, C. (1999). Psychopathy across cultures: North America and Scotland compared. Journal of Abnormal Psychology, 108, 58-68.

Cooke, D. J., Michie, C., Hart, S. D. y Clark, D. (2005). Assessing psychopathy in the UK: Concerns about cross-cultural generalisability. British Journal of Psychiatry, 186, 335-341. doi:10.1192/bjp.186.4.335.

Cooke, D. J., Michie, C., Hart, S. D. y Clark, D. A. (2004). Reconstructing psychopathy: Clarifying the significance of antisocial and socially deviant behavior in the diagnosis of psychopathic personality disorder. Journal of Personality Disorders, 18, 337-357. doi:10.1521/ pedi.18.4.337.40347.

Dolan, M. y Vollm, B. (2009). Antisocial personality disorder and psychopathy in women: A literature review on the reliability and validity of assessment instruments. International Journal of Law and Psychiatry, 32, 2-9. doi:10.1016/j.ijlp.2008.11.002.

Florez, G., Casas, A., Kreis, M. K., Forti, L., Martinez, J., Fernandez, J.,... Cooke, D. J. (2015). A prototypicality validation of the Comprehensive Assessment of Psychopathic Personality (CAPP) Model Spanish Version. Journal of Personality Disorders, 29, 707-718. doi:10.1521/ pedi_2014_28_167.

Gray, N. S. y Snowden, R. J. (2016). Psychopathy in women: Prediction of criminality and violence in UK and USA psychiatric patients resident in the community. 
Psychiatry Research, 237, 339-343. doi:10.1016/j.psychres.2016.01.014.

Guay, J. P., Knight, R. A., Ruscio, J. y Hare, R. D. (2018). A taxometric investigation of psychopathy in women. Psychiatry Research, 261, 565-573. doi:10.1016/j.psychres.2018.01.015.

Hare, R. D., Clark, D., Grann, M. y Thornton, D. (2000). Psychopathy and the predictive validity of the PCL-R: An international perspective. Behavioral Sciences $\mathcal{E}$ the Law, 18, 623-645. doi:10.1002/1099-0798(200010)18:5<623:: aid-bsl409>3.0.co;2-w.

Kreis, M. K., Cooke, D. J., Michie, C., Hoff, H. A. y Logan, C. (2012). The Comprehensive Assessment of Psychopathic Personality (CAPP): Content validation using prototypical analysis. Journal of Personality Disorders, 26, 402-413. doi:10.1521/pedi.2012.26.3.402.

Lenzenweger, M. F., Lane, M. C., Loranger, A. W. y Kessler, R. C. (2007). DSM-IV personality disorders in the National Comorbidity Survey Replication. Biological Psychiatry, 62, 553-564. doi:10.1016/j.biopsych.2006.09.019.

Nicholls, T. L., Ogloff, J. R., Brink, J. y Spidel, A. (2005). Psychopathy in women: a review of its clinical usefulness for assessing risk for aggression and criminality. Behavioral Sciences E $\mathcal{E}$ the Law, 23, 779-802. doi:10.1002/bsl.678.

Rial, A., Burkhart, G., Isorna, M., Barreiro, C., Varela, J. y Golpe, S. (2019). Cannabis use among adolescents: Risk pattern, implications and possible explanatory variables. Adicciones, 31, 64-77. doi:10.20882/adicciones.1212.
Sellbom, M., Cooke, D. J. y Hart, S. D. (2015). Construct validity of the Comprehensive Assessment of Psychopathic Personality (CAPP) concept map: Getting closer to the core of psychopathy. International Journal of Forensic Mental Health, 14, 172-180. doi:10.1080/14999013.2015 .1085112 .

Thomson, N. D. (2020). An exploratory study of female psychopathy and drug-related violent crime. Journal of Interpersonal Violence, 35, 794-808. doi:10.1177/0886260517690876.

Verona, E., Bresin, K. y Patrick, C. J. (2013). Revisiting psychopathy in women: Cleckley/Hare conceptions and affective response. Journal of Abnormal Psychology, 122, 1088-1093. doi:10.1037/a0034062.

Vicens, E., Tort, V., Dueñas, R. M., Muro, Á., Pérez-Arnau, F., Arroyo, J. M.,... Sarda, P. (2011). The prevalence of mental disorders in Spanish prisons. Criminal Behaviour and Mental Health, 21, 321-332. doi:10.1002/cbm.815.

World Medical Association. (2013). World Medical Association Declaration of Helsinki: Ethical principles for medical research involving human subjects. JAMA, 310, 2191-2194. doi:10.1001/jama.2013.281053.

Wynn, R., Hoiseth, M.H. y Pettersen, G. (2012). Psychopathy in women: Theoretical and clinical perspectives. International Journal of Women's Health, 4, 257-263. doi:10.2147/ijwh.S25518. 